\title{
A NEW EXPRESSION FOR UMBRAL OPERATORS AND POWER SERIES INVERSION
}

\author{
A. M. GARSIA AND S. A. JONI
}

\begin{abstract}
It is shown here the compositional inversion of formal power series has a natural formulation in terms of umbral operators. This fact is used to derive a new proof of the Lagrange inversion theorem by operator theoretic methods.
\end{abstract}

Introduction. The main object of this paper is to show that the theory of umbral operators as developed in [1] offers a natural setting for the study of formal power series inversion. A by-product of this idea is perhaps one of the most elementary approaches to the Lagrange inversion theorem in any number of variables.

However, we shall restrict ourselves here to the one-dimensional case leaving the higher-dimensional formulas, as well as some of the applications, to a forthcoming publication.

This paper is divided into two sections. In the first section we recall some of the basic ingredients of the theory of polynomials of binomial type as developed in [1]. In the second section we obtain a new expression for umbral operators which is, in a sense, "dual" to the Lagrange inversion formula. From this expression, several forms of Lagrange's theorem can be immediately derived.

We are indebted to G.-C. Rota for stimulating conversations and encouragement. Indeed, it was during one of our endless discussions with him that the idea developed to put the umbral calculus to such a use.

1. Basics on the theory of umbral operators. Our notation follows very closely that adopted in [1] and the reader would do well to read that paper for a more complete background.

We shall deal with polynomial operators, namely linear maps of polynomials into polynomials. Here, we shall work with polynomials in $\mathscr{F}[x]$, that is, polynomials in the variable $x$ with coefficients in a given field $\mathscr{F}$ of characteristic zero. For sake of simplicity the reader may restrict $\mathscr{F}$ to be the real number field.

This given, we shall express all our formulas by means of the following basic operators:

(1) $L_{0}=$ Evaluation at 0 :

Received by the editors November 23, 1976.

AMS (MOS) subject classifications (1970). Primary 05A15; Secondary 47E05. 


$$
L_{0} x^{n}= \begin{cases}1 & \text { if } n=0 \\ 0 & \text { if } n \geqslant 1\end{cases}
$$

(2) $D=$ Differentiation: $D x^{n}=n x^{n-1}$,

(3) $\underline{x}=$ Multiplication by $x: \underline{x} x^{n}=x^{n+1}$.

Given a formal power series $\Phi(u)=\sum_{\nu=0}^{\infty} \Phi_{\nu} u^{\nu}$ with coefficients in $\mathscr{F}$, we let $\Phi(D)$ denote the polynomial operator

$$
\Phi(D)=\sum_{\nu=0}^{\infty} \Phi_{\nu} D^{\nu}
$$

That is,

$$
\Phi(D) x^{n}=\sum_{\nu=0}^{n} \Phi_{\nu} n(n-1) \cdots(n-\nu+1) x^{n-\nu} .
$$

It can be shown (see [1]) that a polynomial operator $Q$ is of form (1.1) if and only if $Q$ commutes with $D$.

One of the basic tools in [1] as well as here is the fundamental fact that two polynomial operators $P$ and $Q$ that commute with $D$ are identical if and only if

$$
L_{0} P=L_{0} Q
$$

This follows from the fact that in this case (1.2) implies

$$
L_{0} e^{y D} P=L_{0} e^{y D} Q \quad \forall y \in \mathscr{F},
$$

from which the equality of $P$ and $Q$ immediately follows.

There are two further operations that play a crucial role. These are the two "Lie" derivatives $\square$ and $\nabla$ which act on a polynomial operator $P$ as follows:

$$
\begin{aligned}
& \text { (a) } \square P=P \underline{x}-\underline{x} P, \\
& \text { (b) } \nabla P=D P-P D .
\end{aligned}
$$

The first of these was extensively used in [1] and [2]. Both $\square$ and $\nabla$ are differentiations and we have

$$
\begin{aligned}
& \text { (a) } \square D^{n}=n D^{n-1} \text {, } \\
& \text { (b) } \nabla \underline{x}^{n}=n \underline{x}^{n-1} \text {. }
\end{aligned}
$$

Note that if $\Phi(u)$ is a formal power series then

$$
\square \Phi(D)=\Phi^{\prime}(D) .
$$

We shall make extensive use of the trivial identity

$$
L_{0} \square P=L_{0} P \underline{x}
$$


valid for every polynomial operator $P$. This was used in [1] in order to express the "Rodriguez" formula of [2] as an identity for umbral operators. 1

The essential fact expressed by (1.5) is that the derivative $\square$ is the "adjoint" of the operator $\underline{x}$ with respect to the "scalar product" $(P, Q)=L_{0} P(D) Q(x)$.

As in [2] a polynomial operator

$$
f(D)=\sum_{\nu=1}^{\infty} f_{\nu} D^{\nu}, \quad f_{1} \neq 0
$$

will be called a $\Delta$-operator. It is easy to see that a polynomial operator $Q$ is of form (1.6) if and only if $Q$ commutes with $D, Q 1=0$ and $Q x \neq 0$.

Given a $\Delta$-operator $f(D)$ we call

$$
U_{f}=\sum_{\nu=0}^{\infty} \frac{\underline{x}^{\nu}}{\nu !} L_{0} f^{\nu}(D)
$$

the "umbral operator" associated to $f(D)$.

The sequence of polynomials $\gamma_{n}(x)=U_{f} x^{n}$ will be called the B.T. sequence associated to $U_{f}$. Note that since $\gamma_{n}(x)=f_{1}^{n} x^{n}+\cdots$, the sequence $\left\{\gamma_{n}\right\}$ is a basis and thus $U_{f}$ is invertible.

We also note that we have the basic identity

$$
D U_{f}=U_{f} f(D)
$$

Taking all this into account it is easy to see that an operator $U$ is of form (1.7) if and only if

(1) $U$ is invertible,

(2) $L_{0} U=L_{0}$,

(3) $U^{-1} D U$ is a $\Delta$-operator.

Indeed, if $U^{-1} D U=f(D)$ then (1.8) holds and the identity

$$
U=\sum_{\nu=0}^{\infty} \frac{\underline{x}^{\nu}}{\nu !} L_{0} f^{\nu}(D)
$$

follows immediately upon multiplying Taylor's formula

$$
I=\sum_{\nu=0}^{\infty} \frac{\underline{x}^{\nu}}{\nu !} L_{0} D^{\nu}
$$

on the right by $U$.

The relevance of all this material to power series inversion comes from the following fundamental fact pointed out in [2], namely:

The product $U=U_{f} U_{g}$ of two umbral operators is equal to the umbral operator associated to the formal power series

${ }^{1}$ See the proof of Theorem 3.3 in [1]. 


$$
f(g(u))=\sum_{\nu=1}^{\infty} f_{\nu}(g(u))^{\nu}
$$

"functional composition" of $f$ and $g$.

To see this note that (1.8) implies that for every formal power series $\Phi(u)$ we have

$$
\Phi(D) U_{f}=U_{f} \Phi(f(D)) .
$$

This given, we first observe that $U=U_{f} U_{g}$ clearly satisfies both (1.9)(1) and (2). Furthermore, the identities $D U_{f}=U_{f} f(D)$ and $D U_{g}=U_{g} g(D)$ (combined with (1.10)) yield

$$
D U_{f} U_{g}=U_{f} U_{g} f(g(D))
$$

It is well known and easy to show that given any formal power series $f(u)=\sum_{v=1}^{\infty} f_{v} u^{v}\left(f_{1} \neq 0\right)$, there exists a unique formal power series $F(u)$ $=\sum_{v=1}^{\infty} F_{\nu} u^{\nu}\left(F_{1} \neq 0\right)$ such that $f(F(u))=F(f(u))=u$. The existence of $F(u)$ follows immediately from the above considerations since the operator $U_{f}^{-1}$ can be easily shown to satisfy the conditions in (1.9). But then if $U_{f}^{-1}=U_{F}$, the fact that $U_{F} U_{f}=I$ immediately yields that $F(u)$ and $f(u)$ are inverses of each other under functional composition.

It is to be noted that in this case (1.10) with $\Phi(D)$ replaced by $F(D)$ gives

$$
F(D) U_{f}=U_{f} D,
$$

and this in turn is equivalent to (1.8).

2. The Lagrange inversion formula. In this section the formal power series $F(u)=\sum_{\nu=1}^{\infty} F_{\nu} u^{\nu}\left(F_{1} \neq 0\right)$ will be considered as given, and $f(u)$ $=\sum_{\nu=1}^{\infty} f_{\nu} D^{\nu}$, the "functional inverse" of $F(u)$, will be considered as unknown.

The Lagrange inversion formula expresses the coefficients of $f(u)$ directly in terms of the coefficients of $F(u)$.

We shall see that in this context this corresponds to obtaining an expression for the umbral operator $U_{f}=U_{F}^{-1}$ directly in terms of the operator $F(D)$.

Since there is no loss of generality we shall write our formal power series $F(u)$ in the form

$$
F(u)=u-G(u)
$$

where

$$
G(u)=\sum_{\nu=2}^{\infty} G_{\nu} u^{\nu} .
$$

Our basic result can be stated as follows:

THEOREM 2.1. The inverse of the operator $U_{F}$ is given by 


$$
U=F^{\prime}(D) \sum_{\nu=0}^{\infty} \frac{G^{\nu}(D)}{\nu !} \underline{x}^{\nu}
$$

Proof. In view of the above considerations, to show this we need only check that

$$
F(D) U=U D
$$

and

$$
L_{0} U=L_{0} \text {. }
$$

Using (2.1), (2.4) can be written in the form $D U-U D=G(D) U$, that is,

$$
\nabla U=G(D) U \text {. }
$$

However, this follows immediately from (1.4)(b). Indeed, since operators which commute with $D$ behave like constants with respect to $\nabla$, we get

$$
\nabla U=F^{\prime}(D) \sum_{\nu=1}^{\infty} \frac{G^{\nu}(D)}{\nu !} \nu \underline{x}^{\nu-1}
$$

from which (2.6) immediately follows.

As for (2.5) we observe that, in view of the basic identity (1.5), it can be written in the form

$$
L_{0} \sum_{\nu=0}^{\infty} \square^{\nu}(1-\square G) \frac{G^{\nu}}{\nu !}=L_{0},
$$

and this immediately follows from the fact that

$$
\sum_{\nu=0}^{\infty} \square^{\nu}(1-\square G) \frac{G^{\nu}}{\nu !}=\sum_{\nu=0}^{\infty}\left\{\square^{\nu} \frac{G^{\nu}}{\nu !}-\square^{\nu+1} \frac{G^{\nu+1}}{(\nu+1) !}\right\}=I .
$$

The following are two important consequences of the above result.

Corollary 2.1 .

$$
U_{F}^{-1}=I+\underline{x} \sum_{\nu=1}^{\infty} \frac{G^{\nu}(D)}{\nu !} \underline{x}^{\nu-1}
$$

Proof. We can write the operator $U$ in (2.3) in the form

$$
\begin{aligned}
U & =\sum_{\nu=0}^{\infty} \frac{G^{\nu}(D)}{\nu !} \underline{x}^{\nu}-\square \sum_{\nu=0}^{\infty} \frac{G^{\nu+1}(D)}{(\nu+1) !} \underline{x}^{\nu} \\
& =\sum_{\nu=0}^{\infty} \frac{G^{\nu}(D)}{\nu !} \underline{x}^{\nu}-\sum_{\nu=0}^{\infty} \frac{G^{\nu+1}(D)}{(\nu+1) !} \underline{x}^{\nu+1}+x \sum_{\nu=0}^{\infty} \frac{G^{\nu+1}(D)}{(\nu+1) !} \underline{x}^{\nu}
\end{aligned}
$$

from which (2.7) follows immediately.

Corollary 2.1. If 


$$
f(u)=u+G(f(u))
$$

where $G(u)=\sum_{\nu=2}^{\infty} G_{\nu} u^{\nu}$, then for any formal power series $\Phi(u)$ we have

$$
\Phi(f(u))=\Phi(u)+\sum_{\nu=1}^{\infty} \frac{1}{\nu !}\left(\frac{d}{d u}\right)^{\nu-1}\left[\Phi^{\prime}(u) G^{\nu}(u)\right] .
$$

Proof. Clearly (2.8) says that $f(u)$ is the functional inverse of $F(u)$ $=u-G(u)$. Thus, using $(1.8)$ we get

$$
L_{0} \Phi(f(D))=L_{0} U_{f} \Phi(f(D))=L_{0} \Phi(D) U_{f} .
$$

Substituting for $U_{f}$ the expression in (2.7) we obtain

$$
\begin{aligned}
L_{0} \Phi(f(D)) & =L_{0} \Phi(D)+\sum_{\nu=1}^{\infty} L_{0} \Phi(D) \underline{\underline{G^{\nu}}(D)} \frac{\underline{x}^{\nu-1}}{\nu !} \\
& =L_{0} \Phi(D)+\sum_{\nu=1}^{\infty} L_{0} \Phi^{\prime}(D) \frac{G^{\nu}(D)}{\nu !} \underline{x}^{\nu-1} \\
& =L_{0}\left[\Phi(D)+\sum_{\nu=1}^{\infty} \frac{1}{\nu !} \square^{\nu-1}\left\{\Phi^{\prime} G^{\nu}(D)\right\}\right] .
\end{aligned}
$$

Our remark about operators commuting with $D$ then yields

$$
\Phi(f(D))=\Phi(D)+\sum_{\nu=1}^{\infty} \frac{1}{\nu !} \square^{\nu-1}\left\{\Phi^{\prime} G^{\nu}(D)\right\},
$$

and this is clearly equivalent to (2.9).

We shall conclude by deriving one of the standard forms of the Lagrange inversion formula.

THEOREM 2.2. Let $f(u)$ be the formal inverse of the formal power series

$$
F(u)=\sum_{\nu=1}^{\infty} F_{\nu} u^{\nu} \quad\left(F_{1} \neq 0\right) .
$$

Then for any formal power series $\Phi(u)$ we have

$$
\left.\Phi(f(u))\right|_{u^{n}}=\left.(1 / n) \Phi^{\prime}(u)(u / F(u)){ }^{n}\right|_{u^{n-1 .}}{ }^{2}
$$

Proof. Again we may assume with loss that $F_{1}=1$ and set

$$
F(u)=u-u H(u) \text {. }
$$

We shall make use of formula (2.7) with $G(u)=u H(u)$. To this end note that

$$
\begin{aligned}
\left.\Phi(f(u))\right|_{u^{n}} & =(1 / n !) L_{0} \Phi(f(D)) x^{n}=(1 / n !) L_{0} U_{f} \Phi(f(D)) x^{n} \\
& =(1 / n !) L_{0} \Phi(D) U_{f} x^{n} .
\end{aligned}
$$

${ }^{2}$ Here $\left.\right|_{u^{n}}$ means "the coefficient of $u^{n}$ ". 
Substituting (2.7) for $U_{f}$ we obtain

$$
\begin{aligned}
& \left.\Phi(f(u))\right|_{u^{n}}=\frac{1}{n !} L_{0} \Phi(D)\left[x^{n}+\underline{x} \sum_{\nu=1}^{\infty} \frac{H^{\nu}(D)}{\nu !} D^{\nu} x^{n+\nu-1}\right] \\
& =\frac{1}{n !} L_{0} \Phi^{\prime}(D)\left[x^{n-1}+\sum_{\nu=1}^{\infty} H^{\nu}(D) \frac{n(n+1) \cdots(n+\nu-1)}{\nu !} x^{n-1}\right] \\
& =\frac{1}{n !} L_{0} \Phi^{\prime}(D) \frac{1}{(1-H(D))^{n}} x^{n-1} \\
& =\left.\frac{1}{n} \Phi^{\prime}(u) \frac{1}{(1-H(u))^{n}}\right|_{u^{n-1}} \quad \text { Q.E.D. }
\end{aligned}
$$

\section{REFERENCES}

1. A. M. Garsia, An exposé of the Mullin-Rota theory of polynomials of binomial type, Linear and Multilinear Algebra 1 (1973), 47-65. MR 47 \# 8321a.

2. R. Mullin and G.-C. Rota, On the theory of binomial enumeration, Graph Theory and Applications, Academic Press, New York, 1970.

Department of Mathematics, University of California at San Diego, la Jolla, CaliFORNIA 92093 